\title{
Opportunistic Fungal Infections in the Epidemic Area of COVID-19: A Clinical and Diagnostic Perspective from Iran
}

\author{
Mohammadreza Salehi (D) Kazem Ahmadikia (D) Hamid Badali (D) \\ Sadegh Khodavaisy (D)
}

Received: 20 April 2020/Accepted: 30 June 2020/Published online: 31 July 2020

(C) Springer Nature B.V. 2020

\begin{abstract}
The coronavirus disease 2019 (COVID19) pandemic emerged in Wuhan, China, in late 2109 , and has rapidly spread around the world. Until May 25, 2020, there were 133,521 confirmed COVID-19 cases and 7359 deaths in Iran. The role of opportunistic fungal infections in the morbidity and mortality of COVID-19 patients remains less defined. Based on our multicenter experiences, we categorized the risks of opportunistic fungal infections in COVID-19 patients in Iran. The COVID-19 patients at high risk included those with acute respiratory distress syndrome, in
\end{abstract}

Handling Editor: Vishnu Chaturvedi.

M. Salehi

Department of Infectious Diseases, School of Medicine, Imam Khomeini Complex Hospital, Tehran University of Medical Sciences, Tehran, Iran

K. Ahmadikia $\cdot$ S. Khodavaisy $(\bowtie)$

Department of Medical Parasitology and Mycology,

School of Public Health, Tehran University of Medical

Sciences, Tehran, Iran

e-mail: sadegh_7392008@yahoo.com

H. Badali

Invasive Fungi Research Center, Communicable Diseases Institute, Mazandaran University of Medical Sciences,

Sari, Iran

\section{H. Badali}

Fungus Testing Laboratory, Department of Pathology and Laboratory Medicine, University of Texas Health Science Center at San Antonio, San Antonio, TX, USA intensive care units, receiving broad-spectrum antibiotics, immunosuppressants or corticosteroid, and supported by invasive or noninvasive ventilation. The patients were most likely to develop pulmonary aspergillosis, oral candidiasis, or pneumocystis pneumonia. Most diagnoses were probable as the accurate diagnosis of opportunistic fungal infections remains challenging in resource-poor settings. We summarize the clinical signs and laboratory tests needed to confirm candidiasis, aspergillosis, or pneumocystosis in our COVID-19 patients.

Keywords COVID-19 · Fungal infections - Critical patients pneumonia $\cdot$ Iran

An enveloped novel coronavirus, severe acute respiratory syndrome coronavirus 2 (SARS-CoV-2), single-stranded RNA betacoronavirus of the family Coronaviridae, has arisen from Wuhan, China, in late 2019 which posed global healthcare and economic threats [1]. Recently, World Health Organization (WHO) named it coronavirus disease 2019 (COVID19) and declared as a pandemic disease. Despite global containment and quarantine attempts, the reports regarding epidemiological and clinical characteristics of the disease are dramatically increased [2-4]. Despite promising preventive measures being taken, commercially there is no antiviral drugs or vaccine approved to prevent or treat the severe COVID-19 
patients. Although numerous common clinical signs of the disease, i.e., dry cough, coryza, sore throat, dyspnea, myalgia, and fatigue, have been reported $[1,5]$, uncommon symptoms and signs have also been reported. Notably, in critical cases, respiratory failure sets in, leading to acute respiratory distress syndrome (ARDS) with multiorgan failure affecting renal and cardiac function and even death [6]. On February 18, 2020, the first cluster of COVID-19 cases reported in Iran. Despite the application of public health measures, i.e., public education, travel restrictions, case management, contact management, school and daycare measures, and social distancing to reduce disease transmission, it is tremendously spread in the human populations throughout the country. Noteworthily, until May 25, 2020, there were 133,521 confirmed COVID-19 cases and 7359 deaths in Iran. Based on our multicenter experiences in the management of COVID-19 patients, we categorized the risks of opportunistic fungal infections in COVID-19 patients in Iran. In our investigations, the COVID-19 patient populations at the highest risk of opportunistic fungal infections are elderly patients suffering from ARDS, hospitalized in intensive care units (ICU), receiving broad-spectrum antibiotics, patients undergoing invasive or noninvasive ventilation, and those undergoing immunosuppressive or corticosteroid therapies. With this background in mind, we aimed to clarify some concerns regarding the SARS-CoV-2 infected patients were most likely to develop pulmonary aspergillosis, oral candidiasis, or pneumocystis pneumonia

Among various factors leading to morbidity and mortality in COVID-19 patients, opportunistic bacterial or fungal infections could deteriorate the status of patients and leading to ARDS [7]. Although it is not clearly understood, patients with severe COVID-19 are at similar risk of invasive fungal infections which has been already known in patients with severe influenza [7, 8], fungal infections in severely ill patients with COVID-19 in China, France, and Germany were diagnosed [7-9]. Notably on the first week of severely ill patients with COVID-19 admission, we observed several cases of putative oropharyngeal candidiasis (OPC), particularly those hospitalized in intensive care units (ICU) with low lymphocyte counts, under plasmapheresis, or total parenteral nutrition (TPN), due to common Candida species curable by fluconazole and nystatin [10]. Moreover, in some critically ill COVID-19 patients with high level of lactate dehydrogenase (LDH), especially those undergoing immunosuppressive or corticosteroid therapies, based on radiological signs, Pneumocystis jiroveci pneumonia (PJP) were suspected. However, our observations were not supported by mycological, immunological and molecular assay. Remarkably, lymphopenia was the main laboratory finding in $85 \%$ of critically ill patients with COVID-19 $[6,11,12]$. Tan et al. suggested lymphopenia might be a prognostic marker in these populations; patients who died due to COVID-19 had significantly lower lymphocyte counts rather than survivors or those with mild disease [13]. Since the lymphocytes play a decisive role in maintaining immune homeostasis and defensive response against microbial invasion throughout the body, it might be hypothesized that inadequate lymphocyte count may be a key factor contributing to secondary fungal infections such as OPC and PJP in COVID-19 patients [7, 14]. However, clinical course and disease progression of COVID-19 is still poorly understood, there is no specific treatment with proven efficacy, and different therapeutic modalities are being performed. Considering treatment strategies in previous outbreaks of Middle East respiratory syndrome (MERS) and severe acute respiratory syndrome (SARS), proven invasive pulmonary aspergillosis were reported only in few cases of probable SARS cases and were associated with concomitant corticosteroid therapy [15]. Nonetheless, corticosteroid therapy, i.e., hydrocortisone, dexamethasone, and methyl prednisolone, may raise the risk of secondary fungal infections in these patients [16]. Interestingly, a systematic review and meta-analysis revealed a higher mortality rate in influenza patients who received corticosteroids, alongside an increased length of stay in intensive care $(p<0.0001)$ and increased rate (RR 2.0, 95\% CI 1.0-3.8; $p=0.04$ ) of secondary bacterial or fungal infections [17]. Thus, it seems that, coronavirus infection itself might not increase the risk for fungal infections, but other risk factors might have. Besides, using broad-spectrum antibiotics, either empirically or targeted therapy for super-infection in patients with severe COVID-19 raises the odds of fungal infections due to endogenous fungi such as Candida species [18, 19]. Meanwhile, it comes as no surprise that frequent antibiotics usage of meropenem or moxifloxacin in severely ill COVID-19 patients are effective against wide spectrum of bacteria, undoubtedly impair the balance of 


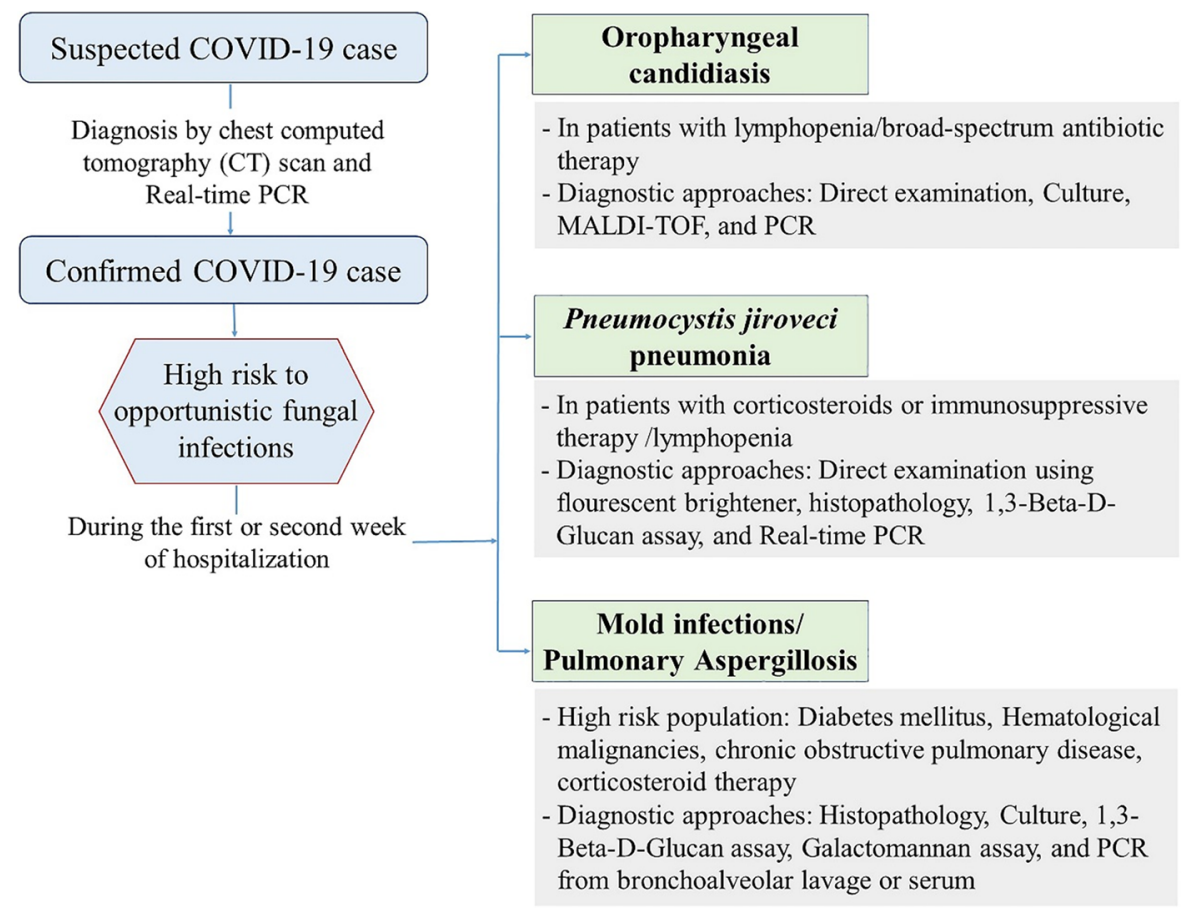

Fig. 1 Clinical and diagnosis data of opportunistic fungal infections in COVID-19 patients

microorganisms resident in mucosal area, leading to further oral health complication [19]. In a study from Chinese hospitals, the usage rate of antibiotics and antifungal agents in patients with severe COVID-19 was $100 \%$ and $39 \%$, respectively [18]. It is well known that ARDS triggered by viral pneumonia, influenza, or cytomegalovirus infections dramatically increases the risk for invasive pulmonary aspergillosis (IPA) even in immunocompetent patients probably due to the overexpression of anti-inflammatory cytokines, dysregulation of T-helper cell differentiation, and impaired cell-mediated immune response [20,21]. Although we are yet to appreciate the incidence and role of IPA as a complication of severe COVID-19 disease in our observations, we have limited data to show how commonly IPA co-infection COVID-19 cases. It seems that more screening studies are highly recommended to evaluate the prevalence of IPA in severely COVID-19 patients [9]. Therefore, the occurrence of IPA and other invasive mold infections are highly probable in COVID-19 patients, particularly in neutropenic patients, those prolongedly use broad-spectrum antibiotics, patients undergoing solid organ and hematopoietic stem cell transplantation (HSCT), those receiving intensive chemotherapy and patients with cystic fibrosis or chronic obstructive pulmonary disease (COPD) [17, 20, 21]. Halo or reversed halo sign was hallmark of pulmonary mold infection in patients with severe influenza; radiological signs might be a valuable tool for diagnosis and management and should take into account for further diagnostic steps in COVID-19 patients [9]. Remarkably, these radiographic patterns have not been previously documented in SARS and MERS patients, but are usually suggestive of IPA $[11,22]$. Li et al. reported 'halo sign' patterns in chest CT examination of nine (18\%) COVID-19 patients [22]. However, the main pathological driver of this manifestation remains unknown, so far. In our opinion, physicians should pay attention to serious challenges associated with OPC, IPA, and PJP in COVID-19 critically ill patients. Nevertheless, due to overlapping clinical symptoms and menace posed by collection of respiratory samples using bronchoscopy that generates aerosolized droplets of SARS-CoV-2 [23], patients with IPA and PJP co-infection are probably underdiagnosed and underreported. Diagnosis of fungal infections in Iran and developing countries with similar infrastructure is based on direct or histopathological microscopy and fungal cultures, but molecular assay, galactomannan, 
1,3- $\beta$-D-glucan antigen testing, and other modern fungal diagnostics assays for further differentiating are rarely available in these region. Clinical and diagnosis data of fungal infections in COVID-19 patients are shown in Fig. 1. Differentiating fungal from bacterial infections based on clinical symptoms, physical findings, radiographic and routine laboratory results is matter of great difficulty. Thus suggesting, if secondary fungal infection is suspected, qualified specimens from the infection site, i.e., sputum or deeply coughed sputum, tracheal aspirates, bronchoalveolar lavage fluid (BAL), or oral swab specimens in case of OPC, should be collected to rule out fungal infections through culture and non-culturebased methods. Furthermore, serial assay of blood 1,3$\beta$-D-glucan, serum and BAL galactomannan testing is crucially recommended to diagnose PJP and IPA alongside routine fungal examinations in suspected cases [24]. In addition, in cases of fever refractory to antibiotic treatment, culture of catheter or peripheral venous blood samples should be timely performed in COVID-19 patients. Because untreated or inadequate treatment of cases increases chances of developing of fungal infection, with attendants significant increase in morbidity and mortality [19, 25]. Therefore, for critically ill patients with COVID-19 who have positive tests for opportunistic fungal infections which may have associated to their death, we have to pay attention to their managements based on national and international guidelines. However, it is imperative to wait the remaining ongoing clinical data to justify fungal complications on COVID-19 patients.

Acknowledgements This study has been funded and supported by Tehran University of Medical Sciences (TUMS); Grant no. 99-1-252-47469.

\section{Compliance with Ethical Standards}

Conflict of interest The authors declare that they have no conflict of interest.

Ethical Approval This study was part of a COVID-19 study that had been approved by the local ethical committees of Tehran University of Medical Sciences under the following ethical code numbers IR.TUMS.VCR.REC.1399.152.

\section{References}

1. Rothan HA, Byrareddy SN. The epidemiology and pathogenesis of coronavirus disease (COVID-19) outbreak. J Autoimmun. 2020;102433.

2. Bedford J, Enria D, Giesecke J, Heymann DL, Ihekweazu C, Kobinger G, et al. COVID-19: towards controlling of a pandemic. Lancet. 2020;395:1015-8.

3. Poli P, Timpano S, Goffredo M, Padoan R, Badolato R. Asymptomatic case of Covid-19 in an infant with cystic fibrosis. J Cyst Fibros. 2020;19:e18.

4. Zhu N, Zhang D, Wang W, Li X, Yang B, Song J, et al. A novel coronavirus from patients with pneumonia in China, 2019. N Engl J Med. 2020;382:727-733.

5. Fishman JA, Grossi PA. Novel coronavirus-19 (COVID-19) in the immunocompromised transplant recipient: \# flatteningthecurve. Am J Transpl. 2020;20(7):1765-1767.

6. Yang X, Yu Y, Xu J, Shu H, Liu H, Wu Y, et al. Clinical course and outcomes of critically ill patients with SARSCoV-2 pneumonia in Wuhan, China: a single-centered, retrospective, observational study. Lancet Respir Med. 2020;8(5):475-481.

7. Gangneux J-P, Bougnoux M-E, Dannaoui E, Cornet M, Ralph ZJ. Invasive fungal diseases during COVID-19: we should be prepared. J Mycol Med. 2020;30(2):100971.

8. Verweij PE, Gangneux J-P, Bassetti M, Brüggemann RJ, Cornely OA, Koehler P, et al. Diagnosing COVID-19-associated pulmonary aspergillosis. Lancet Microbe. 2020;1:e53-5.

9. Alanio A, Delliere S, Fodil S, Bretagne S, Megarbane B. High prevalence of putative invasive pulmonary aspergillosis in critically ill COVID-19 patients. medRxiv. 2020.

10. Salehi M, Ahmadikia K, Mahmoudi S, Kalantari S, Jamalimoghadam Siahkali S, Izadi A, et al. Oropharyngeal candidiasis in hospitalized COVID-19 Patients from Iran: Species identification and antifungal susceptibility pattern. Mycoses. 2020.

11. Huang C, Wang Y, Li X, Ren L, Zhao J, Hu Y, et al. Clinical features of patients infected with 2019 novel coronavirus in Wuhan, China. Lancet. 2020;395(10223):497-506.

12. Ruan Q, Yang K, Wang W, Jiang L, Song J. Clinical predictors of mortality due to COVID-19 based on an analysis of data of 150 patients from Wuhan, China. Intensive Care Med. 2020;46:846-8.

13. Tan L, Wang Q, Zhang D, Ding J, Huang Q, Tang Y-Q, et al. Lymphopenia predicts disease severity of COVID-19: a descriptive and predictive study. Signal Transduct Target Therapy. 2020;5(1):1-3.

14. Ceballos-Salobreña A, Gaitán-Cepeda LA, Ceballos-Garcia L, Lezama-Del VD. Oral lesions in HIV/AIDS patients undergoing highly active antiretroviral treatment including protease inhibitors: a new face of oral AIDS? AIDS Patient Care and STDs. 2000;14(12):627-35.

15. Shang L, Zhao J, Hu Y, Du R, Cao B. On the use of corticosteroids for 2019-nCoV pneumonia. Lancet. 2020;395(10225):683-4.

16. Lim SY, Bolster MB. Corticosteroids. In: Neurorheumatology. Springer; 2019. p. 261-7. 
17. Ni Y-N, Chen G, Sun J, Liang B-M, Liang Z-A. The effect of corticosteroids on mortality of patients with influenza pneumonia: a systematic review and meta-analysis. Crit Care. 2019;23(1):99.

18. Du R-H, Liu L-M, Yin W, Wang W, Guan L-L, Yuan M-L, et al. Hospitalization and critical care of 109 decedents with COVID-19 pneumonia in Wuhan, China. Ann Am Thorac Soc. 2020;17(7):839-846.

19. Laudenbach JM, Epstein JB. Treatment strategies for oropharyngeal candidiasis. Expert Opin Pharmacother. 2009; 10(9):1413-21.

20. McCullers JA. The co-pathogenesis of influenza viruses with bacteria in the lung. Nat Rev Microbiol. 2014;12(4):252-62.

21. Cunha C, Gonçalves SM, Duarte-Oliveira C, Leite L, Lagrou K, Marques A, et al. IL-10 overexpression predisposes to invasive aspergillosis by suppressing antifungal immunity. J Allergy Clin Immunol. 2017;140(3):867-70.

22. Li Y, Xia L. Coronavirus disease 2019 (COVID-19): role of chest CT in diagnosis and management. Am J Roentgenol. 2020;214:1280-6.
23. Wahidi MM, Shojaee S, Lamb CR, Ost D, Maldonado F, Eapen $\mathrm{G}$ et al. The Use of bronchoscopy during the COVID19 pandemic: CHEST/AABIP Guideline and Expert Panel Report. Chest. 2020.

24. Lahmer T, Da Costa CP, Held J, Rasch S, Ehmer U, Schmid $\mathrm{RM}$, et al. Usefulness of 1, 3- $\beta$-D-glucan detection in nonHIV immunocompromised mechanical ventilated critically Ill patients with ARDS and suspected pneumocystis jirovecii pneumonia. Mycopathologia. 2017;182(7-8):701-8.

25. Chaturvedi V, Bouchara J-P, Hagen F, Alastruey-Izquierdo A, Badali H, Bocca AL, et al. Eighty years of Mycopathologia: a retrospective analysis of progress made in understanding human and animal fungal pathogens. Berlin: Springer; 2018.

Publisher's Note Springer Nature remains neutral with regard to jurisdictional claims in published maps and institutional affiliations. 